\section{Radioprotection of Mice by Hypoxia and Chemical Agents}

THe prevalence and magnitude of the effects of oxygen on radiosensitivity have been reviewed by Gray ${ }^{1}$. No other chemical moiety has been reported to exert a greater influence during the period of irradiation. Many investigators accept this 'oxygen effect' as the principal mechanism of radioprotection, and tend to relate the action of all chemical protectants to a simple reduction of oxygen tension. However, observations of the effect of varying oxygen tensions on the radioprotective action of thiols are controversial. For example, breathing oxygen under pressure during the period of irradiation tends to antagonize the protective action of cysteamine and cysteine in rats $^{2}$. In contrast, cysteamine itself appears to increase oxygen tension in the spleen and bone marrow ${ }^{3}$, thus indicating that its protection is not mediated by hypoxia of these radiosensitive tissues. Measuring oxygen tension in tissue by the use of microelectrodes presents numerous problems and the variable results may explain the foregoing observations. Recent work by Vergroesen ${ }^{4}$ demonstrated cysteamine protects mammalian cells against radia. tion even in the presence of complete anoxia, and suggests that the same may be true for mammals. Previous attempts to study the combined effect of low oxygen tension and thiols in the radioprotection of mice were conducted with less than optimal hypoxia ${ }^{5}$. We have reexamined the problem by comparing the 30-day survival of mice irradiated while breathing 5 per cent oxygen, to those irradiated while protected by combinations of hypoxia and chemical agents.

Six weok old female mice (Bagg Swiss), weighing 20 $22 \mathrm{~g}$, were used. Equal numbers of control mice were irradiated simultaneously with each treated group and thereafter housed jointly. Irradiations were accomplished with a cobalt-60 'Gammacell-220'6 at about 1,800 r./min. Four mice at a time were exposed in a perforated 'Plexiglass' dish of doughnut shape, which rotated twice a minute within a cylindrical field of cobalt-60. Hypoxia was induced by forcing the mice to breathe a mixture of 5 per cent oxygen and 95 per cent nitrogen for 90 sec before, and throughout the irradiation period. The gas mixture flowed at a rate of 61 ./min into an irradiation chamber of about 3,000 c.c. volume. Convulsions were prevented by the intraperitoneal administration of 25 $\mathrm{mg} / \mathrm{kg}$ of 'Nembutal' $5 \mathrm{~min}$ prior to gassing. The principles of laboratory animal care as promulgated by the National Society for Medical Research were observed.

The 30-day $L D_{50}$ values for mice irradiated while breathing 5 per cent oxygen, and while breathing air, are presented in Table 1. Their wide divergence illustrates the radioprotective effect of tissue hypoxia. The values were derived in the usual way by plotting the probitper cent mortality versus the logarithm of the dose. Comparing the $L D_{50}$ values for hypoxia and air indicates that hypoxia, as produced in our experiments, reduced the effectiveness of radiation by a factor of $2 \cdot 5$. This dosereduction factor agrees favourably with those established for the relative sensitivities of numerous plant and animal cell systems irradiated aerobically and anaerobically ${ }^{7}$. In addition, it is identical to the dose reduction found by Wright ${ }^{8}$ in young female Swiss mice forced to breathe 100 per cent nitrogen during irradiation. The foregoing correlations, and the clinical appearance of the mice, support the assumption that we have achieved a most favourable degree of intercellular hypoxia.

Table 1. Protective Effect of Hypoxia on Mice exposed to Lethal

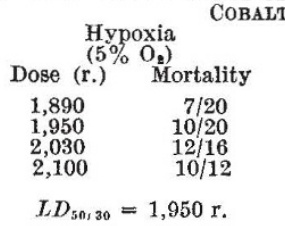

\begin{tabular}{cc}
\multicolumn{2}{c}{$\begin{array}{c}\text { Controls } \\
\text { (air) }\end{array}$} \\
Dose (r.) & Mortality \\
720 & $1 / 12$ \\
760 & $4 / 12$ \\
780 & $7 / 12$ \\
820 & 912 \\
840 & $10 / 12$ \\
$L D_{50,30}=780 \mathrm{r}$.
\end{tabular}

Table 2. Thirty-day Survival Data for Mioe Irradiated while PROTECTED BY HYPOXIA, AND BY HYPOXIA PLUS CHEMICAL AGENTS

Agent
Hypoxia, controls
Hypoxia, plus
p-Aminopropiophenone
p-Aminopropiophenone
5-Hydroxytryptamine
5-Hydroxytryptamine
Dimethylsulphoxide
Diethyldithiocarbamate
Cysteamine
Cysteamine
Cysteamine
Cystamine
Cystamine
S,2-Aminoethyliso-
thiuronium

\begin{tabular}{ccc} 
& \multicolumn{2}{c}{ Intraperito } \\
administrat \\
prior to radi \\
$\begin{array}{c}\text { Cobalt-60 } \\
\text { (r.) }\end{array}$ & $\begin{array}{c}\text { Dose } \\
\text { (mg/kg) }\end{array}$ & T \\
(m) \\
2,300 & & \\
2,400 & \\
2,500 & \\
2,300 & 30 \\
2,400 & 30 \\
2,300 & 75 \\
2,400 & 75 \\
2,400 & 8,000 \\
2,400 & 800 \\
2,300 & 150 \\
2,400 & 150 \\
2,500 & 150 \\
2,400 & 125 \\
2,500 & 125 \\
& \\
& \\
2,400 & 75
\end{tabular}

2,400

75 A

Survival data for mice protected by hypoxia, and by a combination of hypoxia and known radioprotective agents are shown in Table 2 . It can be seen that $2,500 \mathrm{r}$. cobalt- 60 radiation is 100 per cent lethal to mice made radioresistant by hypoxia, and that $2,400 \mathrm{r}$. is the $L D_{99 / 30}$. Our statistics indicate that a 30 per cent difference in survival is significant between groups of animals exposed to 2,400 r. Any survival at 2,500 r. is significant. p-Aminopropiophenone and 5-hydroxytryptamine, both thought to operate through oxygen-dependent pathways ${ }^{1}$, do not add to the protection offered by simple hypoxia. Dimethylsulphoxide and diethyldithiocarbamate also fail to significantly increase the effectiveness of low oxygen tension. However, the thiol agents-cysteamine, cystamine and $S, 2$-aminoethylisothiuronium (AET) - consistently add to protection resulting from hypoxia. Thiols are much less effective against $L D_{100}$ radiation in the hypoxic animal than in the animal breathing air. It is probable that a large portion of their radioprotective activity can be explained by decreased oxygen tension. The significant remainder may also involve the 'oxygen effect', but substantiation awaits the development of a technique for accurately measuring intracellular oxygen levels.

AET, unlike eysteamine, is poorly tolerated by the hypoxic mouse. Table 2 indicates the use of a tolerated dose of AET administered 30 min prior to radiation. Larger doses of chemical, or shorter time intervals, could not be evaluated because acute respiratory failure resulted in excess lethality. Zins ${ }^{9}$ has shown that AET inhibits mitochondrial enzyme activity and results in a build-up of reduced diphosphopyridine nucleotide. This toxic manifestation may explain the foregoing intolerance, and also the loss of protection by AET when animals are forced to breathe oxygen under pressure during radiation ${ }^{2}$.

Our findings support the belief that radioprotection of mice by $p$-aminopropiophenone, 5-hydroxytryptamine, and probably diethyldithiocarbamate and dimethylsulphoxide, is associated with hypoxia of radiosensitive tissues. The action of thiol agents is also concerned with intercellular hypoxia, but hypoxia does not explain the entire radioprotective action. It is probable that metabolic factors, which may be oxygen-dependent, account for the balance of the action by radioprotective thiols.

WILLIAM E. Rothe

Marie M. GrenaN

SHIRLEY M. WILSON

Walter Reed Army Institute of Research, Washington, D.C. ${ }^{1}$ Gray, L. H., Proc. Conf. Res. Radiotherapy of Cancer (N.Y. American

${ }^{2}$ van den Brenk, H., Intern. J. Rad. Biol., 4, 379 (1962).

${ }^{3}$ van der Meer, C., Nature, 189, 588 (1961).

- Vergroesen, A., Nature, 194, 100 (1962).

${ }^{5}$ Devik, F., Acta Radiol., 44, 243 (1955).

Rice, F., Indust. Eng. Chem., 52, No. 5, 47, A (1960).

${ }^{7}$ Gray, L., Rad. Res., Supp. 1, 73 (1959).

${ }^{s}$ Wright, E., Rad. Res., 13, 649 (1960).

' Zins, G., Toxicol. App. Pharmacol, 1, 8 (1959). 